\title{
TRADITIONAL VERSUS NON-TRADITIONAL REINSURANCE IN A DYNAMIC SETTING
}

\author{
Nicole Bäuerle \\ University of Hannover
}

\begin{abstract}
We consider a stochastic risk reserve process whose risk exposure can be controlled dynamically by applying proportional reinsurance and by issuing CAT Bonds. The CAT Bond payments are only partly correlated with the insurers losses. The aim is to minimize the probability of ruin. Using a two-dimensional diffusion approximation we obtain a controlled diffusion problem which can be solved explicitly with the help of the HJB equation. We present some numerical results and discuss to which extend the proportional reinsurance can be replaced by issuing CAT Bonds.

Key words: Optimal control, Hamilton-Jacobi-Bellman equation, diffusion approximation, proportional reinsurance, Catastrophe bonds.
\end{abstract}

\section{Introduction}

In recent years there has been discussions about strategies to transfer part of the insurance risk to the financial market. This idea has mainly been motivated by the hope to open new capacity for the reinsurance market. In our paper we pick a specific new product and investigate whether or not it is suitable to substitute a classical reinsurance product like proportional reinsurance. The analysis is done in a dynamic setting. Nell/Richter (2001) treat this question in a static demand theory environment. As in their paper we focus our comments on a certain type of insurance securitization, namely the issuance of Catastrophe Bonds (CAT Bonds).

A CAT Bond is a risk transfer instrument between the insurer and investor. The investor provides a certain amount of money for which the insurance company offers a coupon payment exceeding the risk-free rate at the end of the coverage period. However, this coupon is only paid if a predefined trigger event does not occur. If the trigger event does occur, then the coupon is not paid and sometimes even part of the invested money is retained by the insurer (for details see Doherty (1997)). There are now different possibilities to define the trigger event. It can be directly based on the insurers losses or on exogeneous indices. The latter case will be treated in this paper. An exogeneous index can for example be an industry loss index or a technical parameter like an earthquake's Richter scale reading or windstorm speed at a certain location. These trigger events if choosen adequately are correlated with the insurers losses. But of course CAT Bonds do not perfectly hedge the risk as traditional reinsurance does. There is still a so-called basis risk. On the positive side, CAT Bonds are in general cheaper than classical reinsurance products (see Froot/O'Connell (1999)).

In order to answer the question to which extend classical reinsurance can be substituted by CAT Bonds, we consider a risk reserve process like in the classical Cramér Lundberg model. We suppose that the management can dynamically control the risk exposure of this process by a mixture of proportional reinsurance and the issuance of CAT Bonds. The 
aim is to minimize the probability of ruin. There are a number of papers considering the question of optimal reinsurance in a dynamic setting for traditional reinsurance contracts and different target functions (among others Højgaard/Taksar (1998), Asmussen et al. (2000), Hipp/Vogt (2001), Schmidli (2001, 2002a,b)). In order to obtain simple structural results for the optimal risk management mix we work with the diffusion approximation of this model. This has already been done in Højgaard/Taksar (1998). Besides these papers there is a growing interest in models with combined reinsurance and investment decisions and in models where the adjustment coefficient is optimized (see for example Schmidli $(2002 \mathrm{a}, \mathrm{b}))$.

The paper is organized as follows: in Section 2 we introduce our model. Section 3 explains the diffusion approximation that is suitable for our setting and the controlled diffusion problem is the subject of Section 4. With the help of the HJB equation we can solve this problem explicitly. For the analysis we have to distinguish the cases of perfect and non-perfect correlation between losses and trigger variable. The case of perfect correlation is not realistic in our model, but treated for the sake of completeness. In Section 5 we briefly discuss the relation between the original model and the diffusion approximation. Numerical results and conclusions are contained in Section 6.

\section{The Model}

We consider a dynamic evolution of the risk reserve of an insurance company like in the Cramér-Lundberg model. This means we suppose that claims arrive according to a Poisson-process $\left(N_{t}\right)$ with intensity $\lambda>0$ and that the claim sizes $X_{1}, X_{2}, \ldots$ form a sequence of non-negative independent and identically distributed random variables. By $m$ we denote the expected claim size and by $s^{2}$ the claim size variance. Since we will make a diffusion approximation later we have to assume that both quantities are finite. The premium income until time $t \geq 0$ is given by $\left(1+\eta_{1}\right) \lambda m t$, where $\eta_{1}>0$ is the safety load of the insurance company. The usual risk reserve is then defined by

$$
R_{t}=x+\left(1+\eta_{1}\right) \lambda m t-\sum_{i=1}^{N_{t}} X_{i}
$$

where $x>0$ is the initial risk reserve. In what follows we denote by $\left(\mathfrak{F}_{t}\right)$ the filtration which is generated by $\left(N_{t}\right)$ and the observable claims.

In order to control the risk reserve, there are two possible risk management tools available: a traditional reinsurance which we suppose to be a proportional reinsurance and a nontraditional way to hedge against risk via CAT Bonds. For the proportional reinsurance we suppose that a proportion $\alpha_{t} \in[0,1]$ can be chosen dynamically over time and $\left(\alpha_{t}\right)$ has to be predictable w.r.t. $\left(\mathfrak{F}_{t}\right)$. This means that the proportion has to be fixed at time $t$ - before we know whether or not a claim at time $t$ arrives. If a claim $X$ arrives the insurance company has to pay $\alpha_{t} X$. The remaining part is covered by the reinsurer. The premium rate for this reinsurance product is given by $\left(1+\eta_{2}\right) \lambda m\left(1-\alpha_{t}\right)$ where $\eta_{2}$ is the safety load of the reinsurer. It is reasonable to assume that $\eta_{2}>\eta_{1}$ because otherwise there exists an arbitrage opportunity for the cedent by giving the whole risk to the reinsurer. On the other hand the insurance company is also able to issue CAT Bonds. We suppose that $\beta_{t} \geq 0$ gives the amount of issued CAT Bonds at time $t \geq 0$. Again $\left(\beta_{t}\right)$ has to be predictable w.r.t. $\left(\mathfrak{F}_{t}\right)$. At claim arrival time points the insurance company will receive an index-linked coverage of fixed size $A>0$ per CAT Bond when an exogenous trigger variable $Y$ which is correlated with the claim size $\mathrm{X}$, reaches a certain level $\bar{y}$. Let $Y_{1}, Y_{2} \ldots$ denote the sequence of trigger variables which we suppose to be independent 
and identically distributed. The dependence relation between the claim size $X_{n}$ and the trigger variable $Y_{n}$ is given by the following conditional probability

$$
p(x)=P(Y \geq \bar{y} \mid X=x) .
$$

It seems to be reasonable to assume that the function $p(x)$ which maps the outcomes of $X$ into $[0,1]$ is increasing. We denote $p:=E[p(X)]$. The cost rate for the CAT Bonds is given by $\lambda A p\left(1+\eta_{3}\right)$ where $\eta_{3}>0$ reflects the issuance costs.

When the management chooses a risk management mix $\pi=\left(\alpha_{t}, \beta_{t}\right)$, the controlled risk reserve process $\left(R_{t}^{\pi}\right)$ is given by

$$
\begin{aligned}
R_{t}^{\pi}= & x+\left(1+\eta_{1}\right) \lambda m t-\left(1+\eta_{2}\right) \lambda m \int_{0}^{t}\left(1-\alpha_{s}\right) d s-\lambda A p\left(1+\eta_{3}\right) \int_{0}^{t} \beta_{s} d s- \\
& \sum_{i=1}^{N_{t}} X_{i} \alpha_{T_{i}}+A \sum_{i=1}^{N_{t}} 1_{\left[Y_{i} \geq \bar{y}\right]} \beta_{T_{i}}
\end{aligned}
$$

where $T_{1}, T_{2}, \ldots$ are the claim arrival time points. Introducing the abbreviations

$$
\theta:=\left(\eta_{2}-\eta_{1}\right) \lambda m, \quad c_{1}:=\left(1+\eta_{2}\right) \lambda m, \quad c_{2}=\lambda A p\left(1+\eta_{3}\right)
$$

and

$$
S_{t}:=\sum_{i=1}^{N_{t}} X_{i}, \quad \hat{S}_{t}:=A \sum_{i=1}^{N_{t}} 1_{\left[Y_{i} \geq \bar{y}\right]}
$$

we obtain

$$
d R_{t}^{\pi}=\left(-\theta+c_{1} \alpha_{t}-c_{2} \beta_{t}\right) d t-\alpha_{t} d S_{t}+\beta_{t} d \hat{S}_{t}
$$

In what follows we assume that $c_{2}<c_{1}$, i.e. the proportional reinsurance is more expensive, however is perfectly correlated with the losses. The survival probability is now defined by

$$
\varphi^{\pi}(x)=P\left(R_{t}^{\pi} \geq 0 \text { for all } t \geq 0 \mid R_{0}^{\pi}=x\right) .
$$

The optimization problem is then to find the risk management mix (if it exists) which maximizes the survival probability

$$
\varphi(x):=\sup _{\pi=\left(\alpha_{t}, \beta_{t}\right)} \varphi^{\pi}(x) .
$$

This piecewise deterministic control problem can be investigated in the same way as similar problems treated e.g. by Schäl (1998) and by Schmidli (2001). However, problems of this kind are quite hard and besides the existence of optimal solutions one gets only a few structural results for the optimal policy. Since our aim is to discuss the relevance of CAT Bonds we pursue a different way. We approximate the problem by a controlled diffusion problem as has been done in Højgaard/Taksar (1998). In this setting we get rather simple explicit solutions (see Section 4). In general it is not clear whether the obtained optimal control of the diffusion problem also behaves well in the original model. This question has been dealt with in Bäuerle (2003) for a model with dividend pay out and reinsurance. We will shortly discuss this question in Section 5 .

\section{Diffusion Approximation in the uncontrolled model}

We consider now the uncontrolled risk reserve process given by

$$
d R_{t}=\left(-\theta+c_{1}-c_{2}\right) d t-d S_{t}+d \hat{S}_{t}
$$


Obviously it holds that

$$
E\left[S_{t}\right]=\lambda m t, \quad \operatorname{Var}\left[S_{t}\right]=\lambda\left(s^{2}+m^{2}\right) t
$$

and

$$
E\left[\hat{S}_{t}\right]=\lambda A p t, \quad \operatorname{Var}\left[\hat{S}_{t}\right]=\lambda A^{2} p t
$$

Thus, the following two processes are martingales with respect to the filtration $\left(\mathfrak{F}_{t}\right)$ for arbitrary $\gamma>0$

$$
M_{t}^{\gamma}:=\frac{1}{\sqrt{\gamma}}\left(\sum_{i=1}^{N_{t \gamma}} X_{i}-\lambda m \gamma t\right) \quad \hat{M}_{t}^{\gamma}:=\frac{1}{\sqrt{\gamma}}\left(A \sum_{i=1}^{N_{t \gamma}} 1_{\left[Y_{i} \geq \bar{y}\right]}-\lambda A p \gamma t\right) .
$$

Of course $\left(M_{t}^{\gamma}\right)$ and $\left(\hat{M}_{t}^{\gamma}\right)$ are not independent. Some easy calculations show

$$
\operatorname{Cov}\left(M_{t}^{\gamma}, \hat{M}_{t}^{\gamma}\right)=\sigma_{1} \sigma_{2} \rho t
$$

with

$$
\sigma_{1}:=\sqrt{\lambda\left(m^{2}+s^{2}\right)}, \quad \sigma_{2}:=A \sqrt{\lambda p}, \quad \rho:=\frac{E[X p(X)]}{\sqrt{p\left(m^{2}+s^{2}\right)}} .
$$

For the following theorem we denote by " $\Rightarrow$ " weak convergence with respect to the Skorohod topology (for definitions and properties see e.g. Whitt (2001), Ethier/Kurtz (1986) or Billingsley (1968)). Our main result of this section is

Theorem 1: (2-dimensional Functional Central Limit Theorem)

For $\gamma \rightarrow \infty$ we obtain $\left(M_{t}^{\gamma}, \hat{M}_{t}^{\gamma}\right) \Rightarrow\left(\sigma_{1} B_{t}, \sigma_{2} \hat{B}_{t}\right)$, where $\left(B_{t}\right)$ and $\left(\hat{B}_{t}\right)$ are two correlated standard Brownian motions with correlation coefficient $\rho$. The parameters $\sigma_{1}, \sigma_{2}$ and $\rho$ are given in (3).

Proof: The proof is similar to the one given in Whitt (2001) Theorem 4.3.5. However, we have to be a little bit more careful here, since the sums contain a random number of terms. The convergence of the one-dimensional marginals is well-known (see e.g. Grandell (1977) or Harrison (1977)). Thus, the marginal processes are tight by Prohorov's Theorem (see e.g. Whitt (2001) Theorem 11.6.1). This implies the tightness of the two-dimensional process $\left(M_{t}^{\gamma}, \hat{M}_{t}^{\gamma}\right)$ (see e.g. Whitt (2001) Theorem 11.6.7). Next, it is not difficult to see that for arbitrary $a, \hat{a} \in \mathbb{R}$ it holds that

$$
\left(a M_{t}^{\gamma}+\hat{a} \hat{M}_{t}^{\gamma}\right) \Rightarrow\left(\sigma_{3} B_{t}\right),
$$

where

$$
\sigma_{3}^{2}:=\lambda\left(a \sqrt{m^{2}+s^{2}}+\rho \hat{a} A \sqrt{p}\right)^{2}+\lambda \hat{a}^{2} A^{2} p\left(1-\rho^{2}\right)
$$

and $\rho$ is given in (3). The limit equals in distribution a mixture of two correlated Brownian motions. Namely,

$$
\left(\sigma_{3} B_{t}\right) \stackrel{d}{=}\left(a \sigma_{1} B_{t}+\hat{a} \sigma_{2} \hat{B}_{t}\right)
$$

where $\sigma_{1}, \sigma_{2}$ are as in (3) and the correlation between $B_{t}$ and $\hat{B}_{t}$ is $\rho$. Thus, the convergence of all finite dimensional distributions of $\left(M_{t}^{\gamma}, \hat{M}_{t}^{\gamma}\right)$ follows with the Cramér-Wold device (see Billingsley (1968) Th. 7.7). This, together with the tightness yields the result.

\section{Remarks:}

a) Observe that $\operatorname{Cov}\left(\sigma_{1} B_{t}, \sigma_{2} \hat{B}_{t}\right)=\sigma_{1} \sigma_{2} \rho t=\operatorname{Cov}\left(M_{t}^{\gamma}, \hat{M}_{t}^{\gamma}\right)$.

b) Suppose $\left(B_{t}\right)$ and $\left(\tilde{B}_{t}\right)$ are two independent standard Brownian motions. $\left(\hat{B}_{t}\right)$ is in distribution equal to $\left(\rho B_{t}+\sqrt{1-\rho^{2}} \tilde{B}_{t}\right)$. 
Now we use Theorem 1 to obtain a diffusion approximation for our model. The data is scaled as follows: the claim arrival intensity $\lambda$ is replaced by $\lambda_{\gamma}:=\lambda \gamma$, the claim sizes $X$ by $X_{\gamma}:=\frac{1}{\sqrt{\gamma}} X$ and the drift $\left(-\theta+c_{1}-c_{2}\right)$ by $\left(-\theta+c_{1}-c_{2}-\lambda m+\lambda A p\right)+\lambda m \sqrt{\gamma}-\lambda A p \sqrt{\gamma}$. Altogether we obtain

$$
R_{t}^{\gamma}=x+\left(-\theta+c_{1}-c_{2}-\lambda m+\lambda A p\right) t-M_{t}^{\gamma}+\hat{M}_{t}^{\gamma} .
$$

Note that $\gamma=1$ is our original model. $\gamma \rightarrow \infty$ yields $\left(R_{t}^{\gamma}\right) \Rightarrow\left(R_{t}\right)$, with

$$
R_{t}=x+\left(-\theta+c_{1}-\lambda m-\left(c_{2}-\lambda A p\right)\right) t-\int_{0}^{t} \sigma_{1} d B_{s}+\int_{0}^{t} \sigma_{2} d \hat{B}_{s}
$$

Introducing the abbreviations

$$
\tilde{\mu}:=c_{1}-\lambda m, \quad \tilde{\nu}:=c_{2}-\lambda A p,
$$

the dynamics of the diffusion risk reserve is given by

$$
d R_{t}=(-\theta+\tilde{\mu}-\tilde{\nu}) d t-\sigma_{1} d B_{t}+\sigma_{2} d \hat{B}_{t} .
$$

\section{The Control Problem}

In a next step we introduce the control again. Since we will investigate the diffusion problem in the rest of the paper, we reuse the notations of Section 2. By $\left(\mathfrak{F}_{t}\right)$ we denote now the filtration generated by $\left(B_{t}\right)$ and $\left(\hat{B}_{t}\right)$.

Admissible controls

An $\left(\mathfrak{F}_{t}\right)$-progressively measurable process $\left(\tilde{\alpha}_{t}\right)$ with values in $[0,1]$ is an admissible control for the proportional reinsurance. Analogously, an $\left(\mathfrak{F}_{t}\right)$-progressively measurable process $\left(\tilde{\beta}_{t}\right)$ with values in $\mathbb{R}_{+}$is an admissible control for the number of issued CAT Bonds. An admissible risk management mix is denoted by $\tilde{\pi}=\left(\tilde{\alpha}_{t}, \tilde{\beta}_{t}\right)$. As we will see, it is more convenient to use the following transformation:

$$
\alpha_{t}:=\tilde{\alpha}_{t} \sigma_{1}, \beta_{t}:=\tilde{\beta}_{t} \sigma_{2}, \quad \mu:=\frac{\tilde{\mu}}{\sigma_{1}}, \nu:=\frac{\tilde{\nu}}{\sigma_{2}} .
$$

Therefore, the transformed admissible controls have to satisfy $\alpha_{t} \in\left[0, \sigma_{1}\right]$ and $\beta_{t} \geq 0$. As before we denote $\pi=\left(\alpha_{t}, \beta_{t}\right)$.

Dynamics of the risk reserve process

The risk reserve under control $\tilde{\pi}=\left(\tilde{\alpha}_{t}, \tilde{\beta}_{t}\right)$ is now given by the following stochastic differential equation

$$
\begin{aligned}
d R_{t}^{\tilde{\pi}} & =\left(-\theta+\tilde{\mu} \tilde{\alpha}_{t}-\tilde{\nu} \tilde{\beta}_{t}\right) d t-\tilde{\alpha}_{t} \sigma_{1} d B_{t}+\tilde{\beta}_{t} \sigma_{2} d \hat{B}_{t} . \\
R_{0}^{\tilde{\pi}} & =x
\end{aligned}
$$

or in terms of the transformed control $\pi=\left(\alpha_{t}, \beta_{t}\right)$, the stochastic differential equation for the risk process is simply

$$
\begin{aligned}
d R_{t}^{\pi} & =\left(-\theta+\mu \alpha_{t}-\nu \beta_{t}\right) d t-\alpha_{t} d B_{t}+\beta_{t} d \hat{B}_{t} . \\
R_{0}^{\pi} & =x .
\end{aligned}
$$


Objective function

The survival probability in this model is given by

$$
\varphi^{\pi}(x)=P\left(R_{t}^{\pi} \geq 0 \text { for all } t \geq 0 \mid R_{0}^{\pi}=x\right)
$$

when we use the control $\pi$. The aim is to find the risk management mix which maximizes the survival probability over all admissible controls

$$
\varphi(x):=\sup _{\pi} \varphi^{\pi}(x) .
$$

This problem has some similarities to the one investigated by Browne (1995). In contrast to our model, however, Browne deals with only one control.

\section{The HJB equation}

We will now solve this control problem via the so-called Hamilton-Jacobi-Bellman (HJB) equation. Note that the quadratic variation of the controlled risk reserve process is given by (see e.g. Karatzas/Shreve (1988))

$$
d<R_{t}^{\pi}>=\left(\alpha_{t}^{2}-2 \alpha_{t} \beta_{t} \rho+\beta_{t}^{2} \rho^{2}+\beta_{t}^{2}\left(1-\rho^{2}\right)\right) d t=\left(\alpha_{t}^{2}+\beta_{t}^{2}-2 \alpha_{t} \beta_{t} \rho\right) d t .
$$

Thus, the Hamilton-Jacobi-Bellman equation of our problem reads

$$
\max _{\substack{0 \leq \alpha \leq \sigma_{1} \\ 0 \leq \beta}}\left\{f_{x}[\mu \alpha-\theta-\nu \beta]+\frac{1}{2} f_{x x}\left[\alpha^{2}+\beta^{2}-2 \alpha \beta \rho\right]\right\}=0 .
$$

The standard solution procedure for the control problem is now as follows (for a background see Fleming/Rishel (1975) or Fleming/Soner (1993)). First we will show that there exists a simple classical solution for the HJB equation, i.e. we have to show that there exists a twice continuously differentiable function $f$ and functions $\alpha^{*}: \mathbb{R}_{+} \rightarrow\left[0, \sigma_{1}\right], \beta^{*}$ : $\mathbb{R}_{+} \rightarrow \mathbb{R}_{+}$such that

$$
\begin{aligned}
0 & =\max _{\substack{0 \leq \alpha \leq \sigma_{1} \\
0 \leq \beta}}\left\{f_{x}(x)[\mu \alpha-\theta-\nu \beta]+\frac{1}{2} f_{x x}(x)\left[\alpha^{2}+\beta^{2}-2 \alpha \beta \rho\right]\right\}= \\
& =f_{x}(x)\left[\mu \alpha^{*}(x)-\theta-\nu \beta^{*}(x)\right]+\frac{1}{2} f_{x x}(x)\left[\left(\alpha^{*}(x)\right)^{2}+\left(\beta^{*}(x)\right)^{2}-2 \alpha^{*}(x) \beta^{*}(x) \rho\right] .
\end{aligned}
$$

$\alpha^{*}$ and $\beta^{*}$ must be admissible feedback controls, i.e. the stochastic differential equation

$$
\begin{aligned}
d R_{t}^{\pi^{*}} & =\left(-\theta+\mu \alpha^{*}\left(R_{t}^{\pi^{*}}\right)-\nu \beta^{*}\left(R_{t}^{\pi^{*}}\right)\right) d t-\alpha^{*}\left(R_{t}^{\pi^{*}}\right) d B_{t}+\beta^{*}\left(R_{t}^{\pi^{*}}\right) d \hat{B}_{t} . \\
R_{0}^{\pi^{*}} & =x
\end{aligned}
$$

must have a unique solution. In a second step we will then verify that the solution of the HJB equation solves our control problem, i.e. $f(x)=\varphi(x)$ and $\pi^{*}=\left(\alpha_{t}^{*}, \beta_{t}^{*}\right)$ with $\alpha_{t}^{*}:=\alpha^{*}\left(R_{t}^{\pi^{*}}\right)$ and $\beta_{t}^{*}:=\beta^{*}\left(R_{t}^{\pi^{*}}\right)$ is an optimal control for our problem.

In what follows we assume that $\mu>\nu$ which reflects the fact that CAT Bonds are cheaper than traditional reinsurance. Since we want to investigate in particular the influence of the correlation $\rho$ between the trigger variable and the insurers losses we have to distinguish between the cases $\rho<1$ and $\rho=1$. Of course $\rho=1$ is not realistic but gives some insight into the mathematical problem.

\subsection{The Case $\rho<1$}

Suppose that $\sigma_{1} \mu>\theta$, otherwise it is easy to see that under every admissible control $\varphi^{\pi}(x)=0$ due to the sample path behavior of the Brownian motion (see e.g. Karatzas/Shreve (1988) Section 2.9). In a first step, we solve the HJB equation (5). In order to simplify the expressions we introduce the following notations: 


$$
d_{1}:=\mu^{2}+\nu^{2}-2 \nu \mu \rho \geq 0, \quad d_{2}:=\mu-\rho \nu \geq 0, \quad d_{3}:=\mu \rho-\nu
$$

and

$$
\hat{\eta}=\hat{\eta}(\theta):=\frac{\sigma_{1}(\mu-\nu \rho)-\theta+\sqrt{\left(\sigma_{1}(\mu-\nu \rho)-\theta\right)^{2}+\sigma_{1}^{2}\left(1-\rho^{2}\right) \nu^{2}}}{\sigma_{1}^{2}\left(1-\rho^{2}\right)} \geq 0 .
$$

Theorem 2: (Solution of the HJB equation)

A solution of the HJB equation is given by $f(x)=1-e^{-\eta x}$ and constant feedback controls $\alpha^{*}(x) \equiv \alpha^{*}, \beta^{*}(x) \equiv \beta^{*}$, where we have to distinguish between the following cases:

a) $\rho<\frac{\nu}{\mu}$ and $\theta<\frac{\sigma_{1} \mu}{2}$ :

$$
\alpha^{*}=\frac{2 \theta}{\mu}, \quad \beta^{*}=0, \quad \eta=\frac{\mu^{2}}{2 \theta} .
$$

b) $\rho<\frac{\nu}{\mu}$ and $\theta \geq \frac{\sigma_{1} \mu}{2}$ :

$$
\alpha^{*}=\sigma_{1}, \quad \beta^{*}=0, \quad \eta=2 \frac{\mu \sigma_{1}-\theta}{\sigma_{1}^{2}} .
$$

c) $\rho \geq \frac{\nu}{\mu}$ and $\theta<\frac{d_{1} \sigma_{1}}{2 d_{2}}$ :

$$
\alpha^{*}=\frac{2 \theta d_{2}}{d_{1}}, \quad \beta^{*}=\frac{2 \theta d_{3}}{d_{1}}, \quad \eta=\frac{d_{1}}{2 \theta\left(1-\rho^{2}\right)} .
$$

d) $\rho \geq \frac{\nu}{\mu}$ and $\frac{d_{1} \sigma_{1}}{2 d_{2}} \leq \theta<\sigma_{1}\left(\mu-\frac{\nu}{2 \rho}\right)$ :

$$
\alpha^{*}=\sigma_{1}, \quad \beta^{*}=\sigma_{1} \rho-\frac{\nu}{\eta}, \quad \eta=\hat{\eta} .
$$

e) $\rho \geq \frac{\nu}{\mu}$ and $\theta \geq \sigma_{1}\left(\mu-\frac{\nu}{2 \rho}\right)$ :

$$
\alpha^{*}=\sigma_{1}, \quad \beta^{*}=0, \quad \eta=2 \frac{\mu \sigma_{1}-\theta}{\sigma_{1}^{2}} .
$$

Proof: For each of the parameter cases we have to check that

$$
\left[\mu \alpha^{*}-\theta-\nu \beta^{*}\right]-\frac{1}{2} \eta\left[\left(\alpha^{*}\right)^{2}+\left(\beta^{*}\right)^{2}-2 \alpha^{*} \beta^{*} \rho\right]=0
$$

and that $\alpha^{*}, \beta^{*}$ are feasible and solve the quadratic programme

$$
(Q P)\left\{\begin{array}{l}
\frac{1}{2} \eta\left[\alpha^{2}+\beta^{2}-2 \alpha \beta \rho\right]-[\mu \alpha-\theta-\nu \beta] \rightarrow \min \\
0 \leq \alpha \leq \sigma_{1} \\
0 \leq \beta
\end{array}\right.
$$

Note that the target function is convex. Thus, this optimization problem can easily be solved with the Lagrange multiplier technique. We will now consider the parameter cases separately:

a) $\rho<\frac{\nu}{\mu}$ and $\theta<\frac{\sigma_{1} \mu}{2}$ :

Plugging in $\alpha^{*}, \beta^{*}$ and $\eta$ verifies (7). To solve the quadratic programme (QP) we consider the corresponding Lagrange function

$$
L\left(\alpha, \beta, y_{1}, y_{2}, y_{3}\right):=\frac{1}{2} \eta\left[\alpha^{2}+\beta^{2}-2 \alpha \beta \rho\right]-[\mu \alpha-\theta-\nu \beta]-y_{1} \alpha+y_{2}\left(\alpha-\sigma_{1}\right)-y_{3} \beta .
$$

The point $\left(\alpha^{*}, \beta^{*}\right)$ is optimal, if we find multipliers $y_{1}^{*}, y_{2}^{*}, y_{3}^{*} \geq 0$ such that the following Karush-Kuhn Tucker conditions are satisfied: 
(i) $\frac{\partial L}{\partial \alpha}\left(\alpha^{*}, \beta^{*}, y_{1}^{*}, y_{2}^{*}, y_{3}^{*}\right)=0$

(ii) $\frac{\partial L}{\partial \beta}\left(\alpha^{*}, \beta^{*}, y_{1}^{*}, y_{2}^{*}, y_{3}^{*}\right)=0$

(iii) $y_{1}^{*} \alpha^{*}=0, y_{2}^{*}\left(\alpha^{*}-\sigma_{1}\right)=0, y_{3}^{*} \beta^{*}=0$

(iv) $0 \leq \alpha^{*} \leq \sigma_{1}, \beta^{*} \geq 0$

It can readily be check that these equations are satisfied if we choose $y_{1}^{*}=0, y_{2}^{*}=$ $0, y_{3}^{*}=\nu-\mu \rho$. Due to our assumption $y_{3}^{*} \geq 0$ is obvious.

b) $\rho<\frac{\nu}{\mu}$ and $\theta \geq \frac{\sigma_{1} \mu}{2}$ :

Note that $\eta \geq 0$ since we assume $\sigma_{1} \mu>\theta$. Plugging in $\alpha^{*}, \beta^{*}$ and $\eta$ verifies (7). In order to solve the quadratic programme we have to choose the multipliers $y_{1}^{*}=0$ and

$$
y_{2}^{*}=\mu-2 \frac{\mu \sigma_{1}-\theta}{\sigma_{1}}, \quad y_{3}^{*}=\nu-2 \rho \frac{\mu \sigma_{1}-\theta}{\sigma_{1}} .
$$

$y_{3}^{*} \geq 0$ is equivalent to $\nu \sigma_{1} \geq 2\left(\mu \sigma_{1}-\theta\right) \rho$. Due to our assumption we have $\sigma_{1} \mu-2 \theta \leq$ 0 . Thus,

$$
\nu \sigma_{1} \geq \sigma_{1} \mu \rho \geq \sigma_{1} \mu \rho+\rho\left(\sigma_{1} \mu-2 \theta\right)
$$

which implies the result. $y_{2}^{*} \geq 0$ is equivalent to $2 \theta \geq \mu \sigma_{1}$ and is hence satisfied.

c) $\rho \geq \frac{\nu}{\mu}$ and $\theta<\frac{d_{1} \sigma_{1}}{2 d_{2}}$ :

After some algebra which is quite lengthy this time we can again verify (7) by inserting the given expressions for $\alpha^{*}, \beta^{*}$ and $\eta$. Since $\rho \geq \frac{\nu}{\mu}$ we have $\beta^{*} \geq 0$ and since $\theta<\frac{d_{1} \sigma_{1}}{2 d_{2}}$ we have $\alpha^{*}<\sigma_{1}$. This parameter setting contains the case where the optimal values for $\alpha$ and $\beta$ are in the interior of the set of admissible actions. Thus, we have to set $y_{1}^{*}=y_{2}^{*}=y_{3}^{*}=0$ and the Karush-Kuhn-Tucker conditions are satisfied.

d) $\rho \geq \frac{\nu}{\mu}$ and $\frac{d_{1} \sigma_{1}}{2 d_{2}} \leq \theta<\sigma_{1}\left(\mu-\frac{\nu}{2 \rho}\right)$ :

Plugging in $\alpha^{*}$ and $\beta^{*}$ in (7) gives us

$$
\eta^{2} \sigma_{1}^{2}\left(\rho^{2}-1\right)+2 \eta\left(\mu \sigma_{1}-\nu \sigma_{1} \rho-\theta\right)+\nu^{2}=0 .
$$

It is not difficult to see that $\eta$ as given in (6) is the only positive zero of this equation. The condition $\beta^{*} \geq 0$ equals $\sigma_{1} \rho \hat{\eta} \geq \nu$. Let us have a look at the mapping $\theta \mapsto \hat{\eta}(\theta)$ as defined in (6). $\hat{\eta}(\theta)$ is decreasing in $\theta$. It can be shown that $\sigma_{1} \rho \hat{\eta}(0)>\nu$ and $\sigma_{1} \rho \hat{\eta}(\theta) \rightarrow 0$ for $\theta \rightarrow \infty$. Thus, there exists a $\hat{\theta}$ with $\sigma_{1} \rho \hat{\eta}(\hat{\theta})=\nu$. Some calculations show that $\hat{\theta}$ is given by $\hat{\theta}=\sigma_{1}\left(\mu-\frac{\nu}{2 \rho}\right)$. Therefore we have $\beta^{*} \geq 0$, if $\theta \leq \sigma_{1}\left(\mu-\frac{\nu}{2 \rho}\right)$. As far as the optimization problem (8) is concerned we have to choose the multipliers $y_{1}^{*}=y_{3}^{*}=0$ and $y_{2}^{*}=\mu-\eta \sigma_{1}\left(1-\rho^{2}\right)-\nu \rho$. Now $y_{2}^{*} \geq 0$ if and only if

$$
\eta \leq \frac{\mu-\nu \rho}{\sigma_{1}\left(1-\rho^{2}\right)}=\frac{\sigma_{1}(\mu-\nu \rho)}{\sigma_{1}^{2}\left(1-\rho^{2}\right)} .
$$

Taking a look at the definition of $\eta$ we see that this is equivalent to

$$
\theta^{2} \geq\left(\sigma_{1}(\mu-\nu \rho)-\theta\right)^{2}+\sigma_{1}^{2}\left(1-\rho^{2}\right) \nu^{2} .
$$

It is now possible to bring this inequality into the form

$$
2 \theta(\mu-\nu \rho) \geq \sigma_{1}\left(\mu^{2}+\nu^{2}-2 \nu \mu \rho\right)
$$

which is satisfied by our assumption.

e) $\rho \geq \frac{\nu}{\mu}$ and $\theta \geq \sigma_{1}\left(\mu-\frac{\nu}{2 \rho}\right)$ :

The verification of (7) is again easy. In order to satisfy the Karush-Kuhn-Tucker conditions we choose $y_{1}^{*}=0$ and

$$
y_{2}^{*}=\mu-2 \frac{\mu \sigma_{1}-\theta}{\sigma_{1}}, \quad y_{3}^{*}=\nu-2 \frac{\mu \sigma_{1}-\theta}{\sigma_{1}} .
$$


The condition $y_{2}^{*} \geq 0$ is equivalent to $\theta \geq \frac{1}{2} \sigma_{1} \mu$ which is satisfied since due to our assumption $\theta \geq \hat{\theta}=\frac{1}{2} \sigma_{1} \mu+\frac{1}{2} \sigma_{1}\left(\mu-\frac{\nu}{\rho}\right)$ and $\mu-\frac{\nu}{\rho} \geq 0$. Finally $y_{3}^{*} \geq 0$ is the same as $\theta \geq \hat{\theta}$ which completes the proof.

\section{Remarks:}

a) Note that all parameter cases are - at least in principle - possible. In particular it holds that

$$
\frac{d_{1} \sigma_{1}}{2 d_{2}}<\sigma_{1}\left(\mu-\frac{\nu}{2 \rho}\right) .
$$

b) Schmidli (2001) has considered among others the diffusion control problem where only proportional reinsurance is allowed. His results (Theorem 1 and Lemma 1) coincide with our findings in case a) and b).

\section{Theorem 3: (Verification Theorem)}

It holds that $\varphi(x)=f(x)$, where $f$ is given in Theorem 2 and $\pi^{*}=\left(\alpha_{t}^{*}, \beta_{t}^{*}\right)$ with $\alpha_{t}^{*} \equiv \alpha^{*}$ and $\beta_{t}^{*} \equiv \beta^{*}$ gives the optimal mix of traditional and non-traditional reinsurance.

Proof: Note that $f(x)=\varphi^{\alpha^{*}, \beta^{*}}(x)$. Hence it suffices to show that $f(x) \geq \varphi(x)$. For the moment we consider only reinsurance strategies with the further restriction that $\beta_{t} \leq M$ for all $t$ and a large constant $M \in \mathbb{R}$. Obviously $\left(\beta_{t}^{*}\right)$ satisfies this constraint if $M$ is large enough. Let now $\pi=\left(\alpha_{t}, \beta_{t}\right)$ be such an arbitrary reinsurance strategy. We have

$$
d R_{t}^{\pi}=\left(-\theta+\mu \alpha_{t}-\nu \beta_{t}\right) d t-\alpha_{t} d B_{t}+\beta_{t} d \hat{B}_{t} .
$$

The ruin time under this control is given by

$$
\tau^{\pi}:=\inf \left\{t \geq 0 \mid R_{t}^{\pi}=0\right\}
$$

Using Itô's formula with $f \in C^{2}$ we obtain

$$
\begin{aligned}
f\left(R_{t \wedge \tau^{\pi}}^{\pi}\right)= & f(x)+\int_{0}^{t \wedge \tau^{\pi}}\left[\left(-\theta+\mu \alpha_{s}-\nu \beta_{s}\right) f^{\prime}\left(R_{s}^{\pi}\right)+\frac{1}{2}\left(\alpha_{s}^{2}+\beta_{s}^{2}-2 \alpha_{s} \beta_{s} \rho\right) f^{\prime \prime}\left(R_{s}^{\pi}\right)\right] d s \\
& -\int_{0}^{t \wedge \tau^{\pi}} f^{\prime}\left(R_{s}^{\pi}\right) \alpha_{s} d B_{s}+\int_{0}^{t \wedge \tau^{\pi}} f^{\prime}\left(R_{s}^{\pi}\right) \beta_{s} d \hat{B}_{s} \\
\leq & f(x)-\int_{0}^{t \wedge \tau^{\pi}} f^{\prime}\left(R_{s}^{\pi}\right) \alpha_{s} d B_{s}+\int_{0}^{t \wedge \tau^{\pi}} f^{\prime}\left(R_{s}^{\pi}\right) \beta_{s} d \hat{B}_{s}
\end{aligned}
$$

where the last inequality follows from the HJB equation (5). Since $\left|f^{\prime}(x)\right| \leq \eta$ and $\alpha_{s} \in\left[0, \sigma_{1}\right], \beta_{s} \in[0, M]$ are bounded the last two stochastic integrals on the righthand side are martingales. Taking expectation on both sides yields $E\left[f\left(R_{t \wedge \tau^{\pi}}^{\pi}\right)\right] \leq f(x)$. Moreover, it holds that

$$
\begin{aligned}
E\left[f\left(R_{t \wedge \tau^{\pi}}^{\pi}\right)\right] & =E\left[f\left(R_{t \wedge \tau^{\pi}}^{\pi}\right) \mid t<\tau^{\pi}\right] P\left(t<\tau^{\pi}\right)+E\left[f\left(R_{t \wedge \tau^{\pi}}^{\pi}\right) \mid t \geq \tau^{\pi}\right] P\left(t \geq \tau^{\pi}\right) \\
& =E\left[f\left(R_{t}^{\pi}\right) \mid t<\tau^{\pi}\right] P\left(t<\tau^{\pi}\right)
\end{aligned}
$$

since $f(0)=0$. The sample paths $R_{t}^{\pi}$ show the following behavior: either $\tau^{\pi}<\infty$ or $R_{t}^{\pi} \rightarrow \infty$ for $t \rightarrow \infty$. Thus, for $t \rightarrow \infty$ the left hand side converges to $\varphi^{\pi}(x)$. Taking the supremum over all bounded strategies $\pi$ yields $\sup _{\pi \text { bounded }} \varphi^{\pi}(x) \leq f(x)$ Since $\beta^{*}<M$ for $M$ large we do not obtain a better value if we allow $\beta^{*}$ to be unbounded. Thus, the inequality holds for all admissible strategies and we obtain $\varphi(x) \leq f(x)$. 


\subsection{The Case $\rho=1$}

Unfortunately the case $\rho=1$ is not a special case of the previous section. We have to consider it separately. The trigger variable for the index-linked payments is here perfectly correlated with the insurers losses. The differential equation for the risk reserve process is then given by

$$
d R_{t}^{\pi}=\left(-\theta+\mu \alpha_{t}-\nu \beta_{t}\right) d t+\left(\beta_{t}-\alpha_{t}\right) d B_{t} .
$$

In order to get a non-trivial problem we assume that $(\mu-\nu) \sigma_{1}<\theta<\sigma_{1} \mu$. If $\theta \geq \sigma_{1} \mu$ it follows that $\varphi(x)=0$, i.e. the survival probability is zero for all admissible controls. If $\theta<(\mu-\nu) \sigma_{1}$ we obtain $\varphi(x)=1$ by setting $\alpha^{*} \equiv \sigma_{1}$ and $\beta^{*} \equiv \sigma_{1}$. This case inherits an arbitrage opportunity: the whole risk can be eliminated by cheaper CAT Bonds. The HJB-equation of this problem reads

$$
\max _{\substack{0 \leq \alpha \leq \sigma_{1} \\ 0 \leq \beta}}\left\{f_{x}[\mu \alpha-\theta-\nu \beta]+\frac{1}{2} f_{x x}[\alpha-\beta]^{2}\right\}=0
$$

We proceed in the same way as before. To ease notation we define

$$
d_{4}:=\sigma_{1}(\nu-\mu)+\theta .
$$

The solution of the HJB equation is given by

Theorem 4: (Solution of the HJB equation)

A solution of the HJB equation is given by $f(x)=1-e^{-\eta x}$ and constant feedback controls $\alpha^{*}(x) \equiv \alpha^{*}, \beta^{*}(x) \equiv \beta^{*}$, where we have to distinguish between the following cases:

a) $\theta<\sigma_{1}\left(\mu-\frac{1}{2} \nu\right)$ :

$$
\alpha^{*}=\sigma_{1}, \quad \beta^{*}=\sigma_{1}-\frac{2 d_{4}}{\nu}, \quad \eta=\frac{\nu^{2}}{2 d_{4}} .
$$

b) $\theta \geq \sigma_{1}\left(\mu-\frac{1}{2} \nu\right)$ :

$$
\alpha^{*}=\sigma_{1}, \quad \beta^{*}=0, \quad \eta=2 \frac{\mu \sigma_{1}-\theta}{\sigma_{1}^{2}} .
$$

Proof: The technique of the proof is the same as for Theorem 2. For each of the parameter cases we have to check that

$$
\left[\mu \alpha^{*}-\theta-\nu \beta^{*}\right]-\frac{1}{2} \eta\left[\left(\alpha^{*}-\beta^{*}\right)^{2}\right]=0
$$

and that $\alpha^{*}, \beta^{*}$ is a minimum point of the quadratic programme

$$
(Q P)\left\{\begin{array}{l}
\frac{1}{2} \eta[\alpha-\beta]^{2}-[\mu \alpha-\theta-\nu \beta] \rightarrow \min \\
0 \leq \alpha \leq \sigma_{1} \\
0 \leq \beta
\end{array}\right.
$$

We consider the two parameter cases separately:

a) $\theta<\sigma_{1}\left(\mu-\frac{1}{2} \nu\right)$ :

Plugging in $\alpha^{*}, \beta^{*}$ and $\eta$ in (11) verifies the first statement. Note that $\beta^{*} \geq 0$ is equivalent to $\theta \leq \sigma_{1}\left(\mu-\frac{1}{2} \nu\right)$. The Lagrange function of the quadratic programme is given by

$$
L\left(\alpha, \beta, y_{1}, y_{2}, y_{3}\right):=\frac{1}{2} \eta[\alpha-\beta]^{2}-[\mu \alpha-\theta-\nu \beta]-y_{1} \alpha+y_{2}\left(\alpha-\sigma_{1}\right)-y_{3} \beta .
$$

As Lagrange multipliers we have to choose $y_{1}^{*}=0, y_{3}^{*}=0$ and $y_{2}^{*}=\mu-\nu \geq 0$. 
b) $\theta \geq \sigma_{1}\left(\mu-\frac{1}{2} \nu\right)$ :

Again, inserting $\alpha^{*}, \beta^{*}$ and $\eta$ in (11) verifies the first statement. The minimization problem (12) is solved by $\alpha^{*}, \beta^{*}$ and the Lagrange multipliers $y_{1}^{*}=0$,

$$
y_{2}^{*}=\eta \mu-\eta^{2} \sigma_{1}, \quad y_{3}^{*}=\eta \nu+\eta^{2} \sigma_{1} .
$$

$y_{3}^{*} \geq 0$ is obvious. $y_{2}^{*} \geq 0$ is equivalent to $\mu-\eta \sigma_{1} \geq 0$ which gives $\mu \sigma_{1} \leq 2 \theta$ by inserting $\eta$. This inequality is satisfied since due to our assumption $2 \theta>\sigma_{1} \mu+\sigma_{1}(\mu-\nu)$ and $\mu-\nu>0$.

The optimality of the solution of the HJB-equation follows as in Theorem 3 .

Theorem 5: (Verification Theorem)

It holds that $\varphi(x)=f(x)$, where $f$ is given in Theorem 4 and $\pi^{*}=\left(\alpha_{t}^{*}, \beta_{t}^{*}\right)$ with $\alpha_{t}^{*} \equiv \alpha^{*}$ and $\beta_{t}^{*} \equiv \beta^{*}$ gives the optimal mix of traditional and non-traditional reinsurance.

\section{Relation to the original model}

An interesting question is whether the simple optimal control obtained for the diffusion control problem in Theorem 3 is in some sense good for our original model. The answer is "Yes" under some additional assumptions. Suppose that the admissible controls for our original model are restricted to feedback controls, i.e. $\alpha_{t}=\alpha\left(R_{t}^{\pi}\right), \beta_{t}=\beta\left(R_{t}^{\pi}\right)$ where $\alpha$ and $\beta$ are Lipschitz-continuous with bounded module. Moreover, $\beta$ is assumed to be bounded. As before we denote by $\gamma>0$ our scaling parameter. For an arbitrary admissible control $\pi^{\gamma}=\left(\alpha_{t}^{\gamma}, \beta_{t}^{\gamma}\right)$, the scaled risk reserve is given by

$$
\begin{aligned}
d R_{t}^{\pi^{\gamma}} & =\left(-\theta+\mu \alpha^{\gamma}\left(R_{t}^{\pi^{\gamma}}\right)-\nu \beta^{\gamma}\left(R_{t}^{\pi^{\gamma}}\right)\right) d t-\alpha^{\gamma}\left(R_{t}^{\pi^{\gamma}}\right) \sigma_{1}^{-1} d M_{t}^{\gamma}+\beta^{\gamma}\left(R_{t}^{\pi^{\gamma}}\right) \sigma_{2}^{-1} d \hat{M}_{t}^{\gamma} \\
R_{0}^{\pi^{\gamma}} & =x
\end{aligned}
$$

The following convergence result for the controlled model can now be proved in the same way as Theorem 3.5 in Bäuerle (2003). Let $A_{t}^{\gamma}:=\int_{0}^{t} \alpha^{\gamma}\left(R_{s}^{\pi^{\gamma}}\right) d s, B_{t}^{\gamma}:=\int_{0}^{t} \beta^{\gamma}\left(R_{s}^{\pi^{\gamma}}\right) d s$.

Theorem 6: Let $\left(\pi^{\gamma}\right)$ be a sequence of controls for $\gamma \rightarrow \infty$. Each sequence of stochastic processes $\left(R_{t}^{\pi^{\gamma}}, A_{t}^{\gamma}, B_{t}^{\gamma}, M_{t}^{\gamma}, \hat{M}_{t}^{\gamma}\right)$ has a convergent subsequence, indexed by $\left(\gamma_{n}\right)$, such that for $n \rightarrow \infty$

$$
\left(R_{t}^{\pi^{\gamma_{n}}}, A_{t}^{\gamma_{n}}, B_{t}^{\gamma_{n}}, M_{t}^{\gamma_{n}}, \hat{M}_{t}^{\gamma_{n}}\right) \Rightarrow\left(R_{t}^{\pi}, \int_{0}^{t} \alpha\left(R_{s}^{\pi}\right) d s, \int_{0}^{t} \beta\left(R_{s}^{\pi}\right) d s, \sigma_{1} B_{t}, \sigma_{2} \hat{B}\right)
$$

where $\left(B_{t}\right)$ and $\left(\hat{B}_{t}\right)$ are two correlated Brownian motions with correlation coefficient $\rho$. The parameters $\sigma_{1}, \sigma_{2}$ and $\rho$ are given in (3). Every limit satisfies $R_{0}^{\pi}=x$ and

$$
d R_{t}^{\pi}=\left(-\theta+\mu \alpha\left(R_{t}^{\pi}\right)-\nu \beta\left(R_{t}^{\pi}\right)\right) d t-\sigma_{1} \alpha\left(R_{t}^{\pi}\right) d B_{t}+\sigma_{2} \beta\left(R_{t}^{\pi}\right) d \hat{B}_{t}
$$

The scaled maximal survival probability is given by

$$
\varphi^{\gamma}(x)=\sup _{\pi^{\gamma}} \varphi^{\pi^{\gamma}}(x)=\sup _{\pi^{\gamma}} P\left(R_{t}^{\pi^{\gamma}} \geq 0 \text { for all } t \mid R_{0}^{\pi^{\gamma}}=x\right) .
$$


Note that for $\gamma=1$ we obtain our original control problem of Section 2. The relation between $\varphi^{\gamma}$ and $\varphi$ is now revealed in the following theorem.

\section{Theorem 7: (Asymptotic optimality)}

a) For all $x \geq 0$ and all controls $\left(\pi^{\gamma}\right)$ it holds that

$$
\limsup _{\gamma \rightarrow \infty} \varphi^{\pi^{\gamma}}(x) \leq \varphi(x) .
$$

b) For the optimal control $\pi^{*}$ of the diffusion model we obtain

$$
\lim _{\gamma \rightarrow \infty} \varphi^{\pi^{*}, \gamma}(x)=\varphi(x)
$$

Proof:

a) Let $\left(\pi^{\gamma}\right)$ be an arbitrary sequence of controls and define $\tau^{\pi^{\gamma}}:=\inf \left\{t \geq 0 \mid R_{t}^{\pi^{\gamma}}<0\right\}$. Then $\varphi^{\pi^{\gamma}}(x)=P\left(\tau^{\pi^{\gamma}}=\infty \mid R_{0}^{\pi^{\gamma}}=x\right)$. Suppose that $\{\gamma\}$ is a subsequence which yields convergence in Theorem 6 . Note that the ruin probability in finite time is a continuous mapping (see e.g. Grandell (1991) Sec. 1.2). Thus, due to the continuous mapping theorem (see e.g. Whitt (2001) Theorem 3.4.1) and the monotonicity of the probability measure we have

$$
\begin{aligned}
\limsup _{\gamma \rightarrow \infty} \varphi^{\pi^{\gamma}}(x) & =\limsup _{\gamma \rightarrow \infty} \lim _{t_{0} \rightarrow \infty} P\left(\tau^{\pi^{\gamma}}>t_{0} \mid R_{0}^{\pi^{\gamma}}=x\right) \leq \\
& \leq \lim _{t_{0} \rightarrow \infty} \limsup _{\gamma \rightarrow \infty} P\left(\tau^{\pi^{\gamma}}>t_{0} \mid R_{0}^{\pi^{\gamma}}=x\right)=\varphi^{\pi}(x) \leq \varphi(x) .
\end{aligned}
$$

b) Under the optimal control $\pi^{*}$ of Theorem 3 the system behaves like a classical ruin model where the convergence of the ruin probability is well known (see e.g. Grandell Sec. $1.2(1991))$.

Theorem 7 in particular implies that if our original problem is close to the diffusion limit, then the diffusion optimal control $\pi^{*}$ is nearly optimal for our problem.

\section{$6 \quad$ Numerical Results and Conclusions}

From Theorem 2 we can conclude that CAT Bonds are never used for reinsurance if the correlation between the trigger variable and the insurers losses is too low $\left(\rho<\frac{\nu}{\mu}\right)$. This seems to be a reasonable behavior. However, it is quite surprising that $\beta^{*}$ is very sensitive with respect to the parameter $\theta$. $\theta$ is a measure for the difference of the premium rates between cedent and reinsurer. In the following figures 1-3 we have plotted the optimal reinsurance mix $\left(\sigma_{1}-\alpha^{*}, \beta^{*}\right)$ as a function of $\theta$. Note that we take $\sigma_{1}-\alpha^{*}$ here, which means that $\sigma_{1}-\alpha^{*}=0$ corresponds to no proportional reinsurance. This seems to be more intuitive than plotting $\alpha^{*}$. We have set the parameters as follows: $\sigma_{1}=\sigma_{2}=1, \mu=3$ and $\nu=1$. In figure 1 we chose $\rho=0.5$, in figure $2, \rho=0.75$ and in figure $3, \rho=0.99$. Thus, we are in case c)-e) of Theorem 2. The optimal quota share for the proportional reinsurance decreases with $\rho$. This can be seen from the formulas as well as from the figures. The amount of issued CAT Bonds increases with the correlation $\rho$ which is again intuitive. However, when we vary $\theta$, we see that the number of issued CAT Bonds first increases and after reaching a maximum decreases again in a nonlinear fashion. Thus, in situations where the premium rate difference is large (which is reflected by large $\theta$ ), 
the instrument of CAT Bonds is also of no use. When the insurance company faces a moderate premium difference then indeed the proportional reinsurance is reduced in favor of the CAT Bonds. In cases of low premium differences, the proportional reinsurance is superior. Figure 3 also contains the case where $\rho=1$. From Section 4 we know that in this case $\sigma_{1}-\alpha^{*}=0$, i.e. we have no proportional reinsurance. If $\theta \leq(\mu-\nu) \sigma_{1}$, the whole risk can be eliminated by CAT Bonds. If $\theta>(\mu-\nu) \sigma_{1}$ again the amount of issued CAT Bonds is decreasing. If $\theta>2.5$ no CAT Bonds are issued.

Of course the relation between the parameters of the diffusion model and the parameters of the original model is quite involved. For example if one increases the cost of proportional reinsurance by increasing $\eta_{2}$, then also $c_{1}$ is increasing which leads to an increasing $\mu$. This however means that the issuance of CAT Bonds becomes profitable for lower correlations $\rho$.

Altogether it seems that CAT Bonds are only of limited value as a substitute for traditional reinsurance. In any case the number of issued CAT Bonds is bounded. The usage of this instrument is reasonable only when the correlation between trigger event and losses is large enough and the insurance company faces a moderate premium rate difference.

\section{References}

[1] Asmussen, S., B. Højgaard, and M. Taksar (2000): Optimal risk control and dividend distribution policies. Example of excess-of loss reinsurance for an insurance corporation. Finance and Stochastics 4, 299-324.

[2] B̈̈Uerle, N. (2003): Approximation of optimal reinsurance and dividend pay-out policies. To appear in Math. Finance.

[3] Billingsley, P. (1968): Convergence of Probability measures. John Wiley \& Sons. New York.

[4] Browne, S. (1995): Optimal investment policies for a firm with random risk process: exponential utility and minimizing the probability of ruin. Math. Operations Res. 20, 937-958.

[5] Doherty, N. (1997): Financial innovation for financing and hedging catastrophe risk. In: Financial risk management for natural catastrophes. Eds. Britton, N. and J. Oliver. Conference Proceedings, Griffith University, Brisbane.

[6] Ethier, S.N., And T.G. Kurtz (1986): Markov processes. New York: John Wiley \& Sons.

[7] Fleming, W.H., And R.W. Rishel (1975): Deterministic and stochastic optimal control. Springer-Verlag, New York.

[8] Fleming, W.H., And H.M. Soner (1993): Controlled Markov processes and viscosity solutions. Springer-Verlag, New York.

[9] Froot, K.A., and P.G.J. O'Connell (1999): The pricing of US catastrophe reinsurance. In The financing of catastrophe risk. Ed. Froot, K.A. University of Chicago Press, Chicago.

[10] Grandell, J. (1977): A class of approximations of ruin probabilities. Scand. Actuarial J., Suppliment 37-52. 
[11] Grandell, J. (1991): Aspects of risk theory. Springer-Verlag, Berlin.

[12] Harrison, J.M. (1977): Ruin problems with compounding assets. Stochastic Processes Appl. 5, 67-79.

[13] Hipp, C., And M. Vogt (2001): Optimal dynamic XL reinsurance. To appear in ASTIN Bulletin.

[14] Højgaard, B., And M. Taksar (1998): Optimal proportional reinsurance policies for diffusion models. Scand. Actuarial J. 2, 166-180.

[15] Karatzas, I., And S.E. Shreve (1988):Brownian motion and stochastic calculus. Springer-Verlag, New York.

[16] Nell, M., AND A. Richter (2001): Catastrophe Index-linked securities and reinsurance as substitutes. Unpublished observation.

[17] SchёL, M. (1998): On piecewise deterministic Markov control processes: control of jumps and of risk processes in insurance. Insurance Math. $\&$ Econom. 22, 75-91.

[18] Schmidur, H. (2001): Optimal proportional reinsurance policies in a dynamic setting. Scand. Actuarial J. 1, 55-68.

[19] Schmidui, H. (2002a): Asymptotics of ruin probabilities for risk processes under optimal reinsurance policies: the large claim case. Unpublished observation.

[20] Schmidui, H. (2002b): Asymptotics of ruin probabilities for risk processes under optimal reinsurance policies: the small claim case. Unpublished observation.

[21] Whitт, W. (2001): Stochastic process limits. Springer-Verlag.

Nicole Bäuerle

Institute for Mathematical Stochastics

University of Hannover

Welfengarten 1

D-30167 Hannover, Germany

e-mail: baeuerle@stochastik.uni-hannover.de 


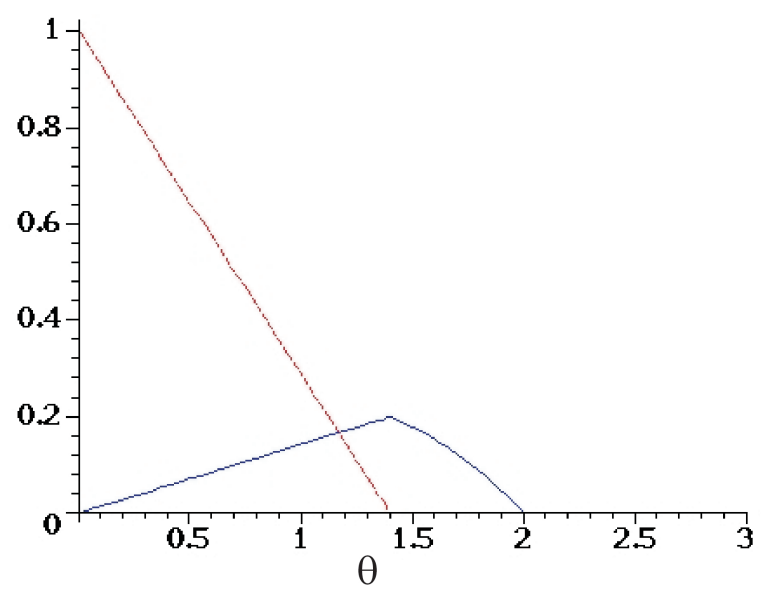

fig 1.: $\sigma_{1}-\alpha^{*}$ (dashed line) and $\beta^{*}$ (solid line) depending on $\theta$ for $\rho=0.5$.

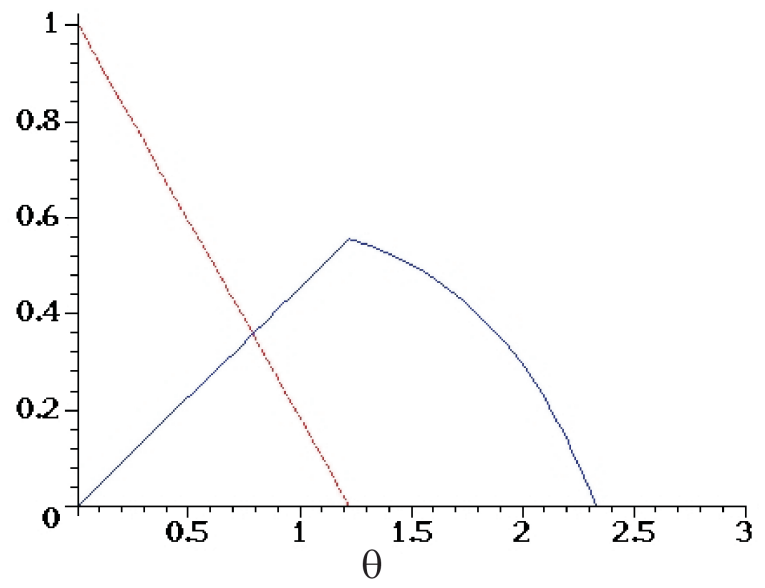

fig 2.: $\sigma_{1}-\alpha^{*}$ (dashed line) and $\beta^{*}$ (solid line) depending on $\theta$ for $\rho=0.75$.

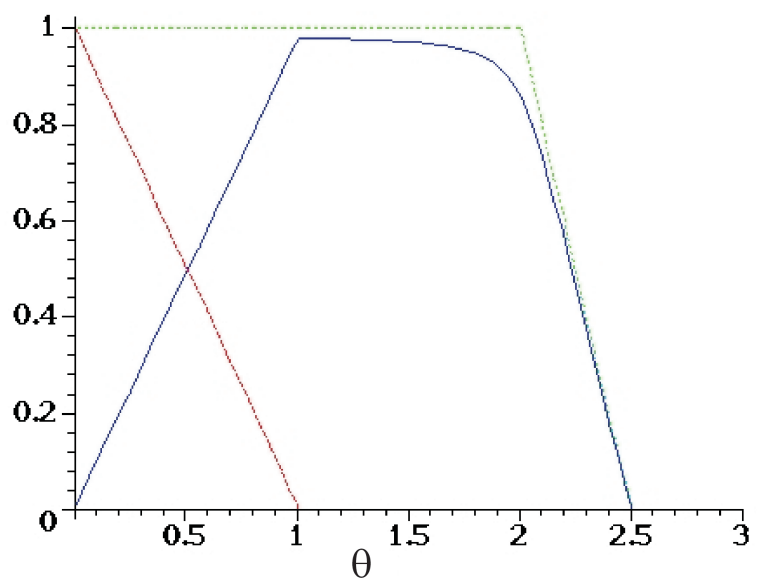

fig 3.: $\sigma_{1}-\alpha^{*}$ (dashed line) and $\beta^{*}$ (solid line) depending on $\theta$ for $\rho=0.99$ and $\beta^{*}$ depending on $\theta$ for $\rho=1$ (dotted line). 